\title{
Ketone-Alcohol Hydrogen Transfer Equilibria: Is the Biooxidation of Halohydrins Blocked?
}

\author{
Fabricio R. Bisogno, ${ }^{[\mathrm{a}]}$ Eduardo García-Urdiales, ${ }^{[\mathrm{a}]}$ Haydee Valdés,${ }^{[\mathrm{b}]}$ Iván Lavandera, ${ }^{[\mathrm{a}]}$ Wolfgang \\ Kroutil, ${ }^{[c]}$ Dimas Suárez, ${ }^{[b]}$ and Vicente Gotor* ${ }^{[a]}$
}

In memoriam of Prof. José Manuel Concellón

\begin{abstract}
In order to ensure the quasiirreversibility of the oxidation of alcohols coupled with the reduction of ketones in a hydrogen transfer (HT) fashion, stoichiometric amounts of $\alpha$ halo carbonyl compounds as hydrogen acceptors have been employed. The reason why these substrates lead to quasi-quantitative conversions has been tacitly attributed to both thermodynamic and kinetic effects. In order to provide a clear rationale for this behavior, we study here the redox equilibrium of a selected series of ketones and 2-propanol by undertaking an approach that combines experimental and theoretical elements. First, the activity of the $R$-specific alcohol dehydrogenase from Lactobacillus brevis (LBADH) with these substrates is studied. The docking
\end{abstract}

of acetophenone/ (R)-1-phenyethanol, and $\alpha$-chloroacetophenone/ $(S)$-2chloro-1-phenylethanol in the active site of the enzyme confirms that there seems not to be any structural reason for the lack of reactivity of halohydrins. This assumption is confirmed by the fact that the corresponding aluminumcatalyzed Meerwein-Ponndorf-VerleyOppenauer (MPVO) reactions afford similar conversions as those obtained with LBADH, evidencing that the observed reactivity is independent from the catalyst employed. While the initial rates of the enzymatic reductions and the IR $v(\mathrm{C}=\mathrm{O})$ values contradict the general belief that electronwithdrawing groups increase the electrophilicity of the carbonyl group, the calculated $\Delta G$ values of the isodesmic redox transformations of these series of ketones/alcohols with 2propanol/ acetone support the thermodynamic control. As a result, a general method to predict the degree of conversion obtained in the HTreduction process of a given ketone based on the IR absorption band of the carbonyl group is proposed, and a strategy to achieve the HT oxidation of halohydrins is also shown.

\section{Keywords: hydrogen transfer • alcohol dehydrogenases • halohydrin • computer chem. • IR}

[a] F. R. Bisogno, Dr. E. García-Urdiales, Dr. I. Lavandera, Prof. Dr. V. Gotor Departamento de Química Orgánica e Inorgánica Instituto Universitario de Biotecnología de Asturias, Universidad de Oviedo

C/ Julián Clavería 8, 33006 Oviedo, Spain

Fax: (+)34985103448

E-mail: vgs@fq.uniovi.es

[b] Dr. H. Valdés, Prof. D. Suárez

Departamento de Química Física y Analítica

Universidad de Oviedo

C/ Julián Clavería 8, 33006 Oviedo, Spain

[c] Prof. W. Kroutil

Department of Chemistry, Organic and Bioorganic Chemistry

University of Graz

Heinrichstrasse 28, $8010 \mathrm{Graz}$, Austria

Supporting information for this article is available on the WWW under http://www.chemeurj.org/ or from the author.

\section{Introduction}

Physicists and chemists have unveiled the factors that control and determine the final concentrations of the species involved in a given transformation. ${ }^{[1]}$ The ability to shift a chemical equilibrium is crucial in Organic Synthesis as it allows the maximization of the yield of the target product(s). Whenever the equilibrium constant does not lead to complete conversion, the tuning of the reaction conditions, such as temperature, pressure, reagents stoichiometry, as well as medium engineering and product(s) removal from the reaction medium can be used to drive the reaction toward the desired direction. ${ }^{[2]}$

The equilibrium constant in redox processes is mainly governed by the difference in oxidation-reduction potentials $\left(\triangle E^{0}\right)$ between reagents. In general, oxidizing or reducing species with much higher potentials than the starting materials are used ${ }^{[3]}$ thus ensuring the completeness of the reaction. However, in some cases this is not 
possible or not desirable due to incompatibilities with other functional groups in the molecule or because of hazardous conditions. The reduction of ketones coupled with the oxidation of alcohols in a hydrogen transfer (HT) fashion is an example ${ }^{[4]}$ of a redox process in which the $\Delta E^{0}$ between the hydrogen donor and the acceptor is not usually large enough to achieve quantitative conversions. Enzymes such alcohol dehydrogenases (ADHs, Scheme $1 \mathrm{~A})^{[5]}$ and metal complexes [Meerwein-Ponndorf-Verley reduction/Oppenauer oxidation (MPVO); Scheme $1 \mathrm{~B}]^{[4]}$ have successfully been employed as catalysts in this kind of transformation. Traditionally, the equilibrium is shifted toward the reduction/oxidation of the ketone/alcohol of interest by sacrificing a small and inexpensive alcohol or ketone, like 2-propanol or acetone (employed as co-substrate) in a huge molar excess: at least 10 equivalents to afford conversions higher than $90 \%$. However, in order to maximize the atom efficiency ${ }^{[6]}$ of this type of process, it is highly desirable to find reaction conditions which allow quasiirreversible hydrogen transfer protocols. ${ }^{[7]}$

We have observed that the biocatalyzed reduction of $\alpha$ haloketones proceeded quasi-irreversibly when coupled with the oxidation of different secondary alcohols. ${ }^{[8]}$ Thus, although ADHs nicely reduced $\alpha$-chloroketones into the corresponding 1-chloro-2alcohols, we did not detect the oxidation of halohydrins like 1chloro-2-octanol and 2-chloro-1-phenylethanol by a library of more than 60 commercial ADHs employing acetone as hydrogen acceptor. ${ }^{[9]}$ As a tentative explanation of this reactivity, it was put forward that the redox potential gap between the $\alpha$ chloroketone/halohydrin pair with respect to the 2-propanol/acetone one is higher than for related non-halogenated pairs. ${ }^{[10]}$ With this regard, it has been shown that halohydrins could additionally be stabilized by the presence of an intramolecular H-bond between the alcohol moiety and the halogen atom, ${ }^{[11]}$ which, on the other hand, could also block the ADH-catalyzed oxidation. Therefore, this behavior seems not to be a particular feature of ADH-catalyzed processes, but rather a general aspect of this type of substrate. Thus, the MPVO-HT (Scheme 1B) of $\alpha$-halogenated carbonyl compounds also proceeds quasi-irreversibly. In particular, chloral (trichloroacetaldehyde), ${ }^{[12]} \quad \alpha$-chloroacetophenone, ${ }^{[13]} \quad \alpha$ bromoacetophenone, ${ }^{[13 a]}$ and 1,1,1-trifluoroacetone ${ }^{[14]}$ have been quantitatively reduced when employed as stoichiometric hydrogen acceptors in the presence of alcohols like 2-propanol by using different $\mathrm{Al}$ or $\mathrm{Zr}$ complexes as catalysts. ${ }^{[15]}$

Ketones bearing electron-withdrawing groups (EWGs) are tacitly regarded as electron-deficient ketones and, hence, can be more readily attacked by nucleophiles (e.g., hydride) than "nonactivated" ketones, which would lead to higher reduction rates. On the other hand, it has also been argued that $\alpha$-halo substituents increase the stability of the corresponding alcohols by destabilizing the partial positive charge formed during their oxidation, ${ }^{[16]}$ thus leading to higher energy transition states for the reverse reaction and hence, to an increase of the activation energy for the oxidation of halohydrins. This aspect is in favor of both a kinetic blockade of the oxidation of this kind of alcohol and the commonly accepted fact that the Oppenauer oxidation of alcohols is under thermodynamic control, i.e. the final composition of the reaction mixture is solely determined by the reduction potentials of the ketones/aldehydes involved. ${ }^{[4]}$

In summary, to explain the conversion in this type of process a variety of kinetic and thermodynamic hypotheses have been put forward. In order to provide an unambiguous rationale for the final conversion of these HT reductions we have studied here the redox equilibrium of a series of selected ketones. The main goals were the correlation of the conversions observed with the catalyst employed and the structure of the substrates, as well as the extent of the influence of kinetics and thermodynamics. To achieve this goal, we undertook a combined experimental and theoretical approach in which, first, several ketone/alcohol complexes bound to the $R$ specific NADPH-dependent alcohol dehydrogenase from Lactobacillus brevis (LBADH) ${ }^{[17]}$ were structurally analyzed. Next, the corresponding aluminum-catalyzed MPVO-HT reactions were carried out to observe the influence of the catalyst in these processes. Finally, the enzymatic initial rates and the IR absorption bands of the carbonyl groups were measured, and ab initio calculations of the ketone/alcohol pairs were performed. As a result, a general method to predict the degree of conversion in the HT-reduction of a given ketone was proposed, and a strategy to achieve the HT oxidation of halohydrins was developed.

\section{Results and Discussion}

\section{LBADH-catalyzed reduction of ketones under HT conditions}

The ketones listed in Table 1 show a wide range of conversions at equilibrium when biocatalytically reduced in the presence of 2 equiv of ${ }^{i} \mathrm{PrOH}(\mathbf{1 a}) .{ }^{[8,18]}$ As can be seen, LBADH reduces acetophenone (2b, entry 1) at approx. 50\% conv. If we use this ketone as reference substrate, the introduction of electronegative groups at the small substituent leads to much higher conversions. Thus, for 2chloroacetophenone (2c, entry 2) the conversion is quantitative and for 2-azido-acetophenone (2d, entry 3 ) close to completion. The same effect, but to a lesser extent, is observed when the substituents are present at the aromatic ring. Thus, for the electron-withdrawing nitro group (2e, entry 4) the conversion increases considerably $(85 \%)$ as compared to acetophenone. Conversely, when an electrondonating group is present at the aromatic ring (2f, entry 5), the equilibrium is shifted toward the opposite direction. 
A)

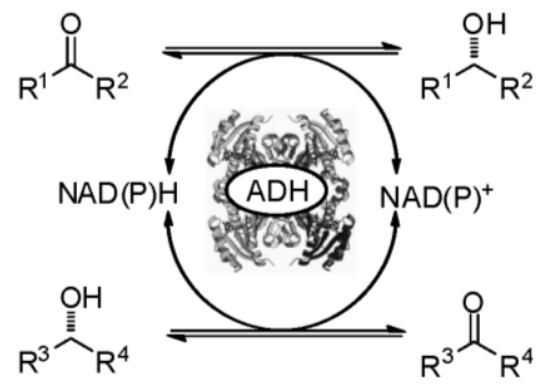

B)

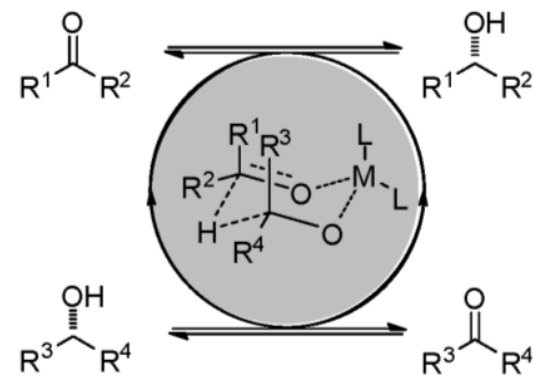

Scheme 1. Reduction of ketones coupled with the oxidation of alcohols catalyzed by: A) ADHs; and B) metal complexes (MPVO).

The employment of aliphatic ketones leads to similar results. Thus, the presence of electronegative groups as substituents of the ketone function increases considerably the conversion at equilibrium, being the chloromethylene group (2h, entry 7) again more efficient than the methoxymethylene one (2g, entry 6). From these results it can be concluded that, as compared to acetophenone, the oxidation of ${ }^{i} \mathrm{PrOH}$ is more efficient when ketones bearing EWGs are used. Moreover, this efficiency seems to be independent of the nature of the ketone (aromatic or aliphatic).

Table 1. Equilibrium of enzymatic reduction of ketones $\mathbf{2 b}-\mathbf{h}$ catalyzed by LBADH employing 2 equiv. of 2-propanol.<smiles>[R]C([R])=O</smiles>

$1 \mathrm{a}$ 2b-h$$
\rightleftharpoons
$$

LBADH<smiles>C1=CC=C1</smiles><smiles>[R]C([OH2+])[18OH]</smiles>

2a

$(R)-\mathbf{1 b}, \mathbf{e}-\mathbf{g}$ (S)-1c-d,h

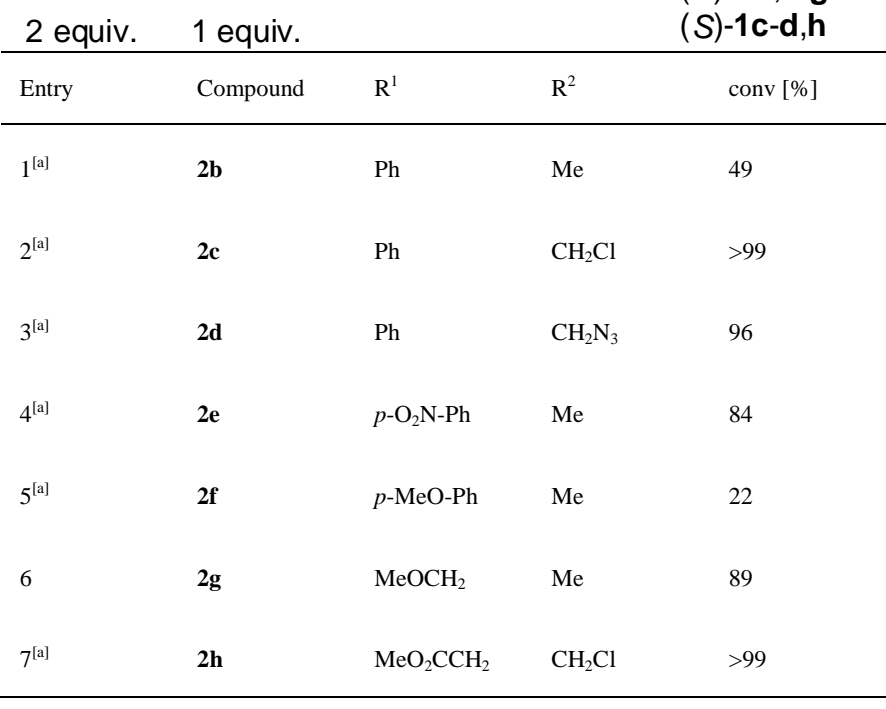

[a] See Reference 8.

\section{Docking of alcohols $(R)-1 b$ and $(S)-1 \mathrm{c}$, and ketones $2 \mathrm{~b}-\mathrm{c}$ to LBADH}

The typical substrates of LBADH are prochiral ketones with a methyl group as small substituent and, conversely to the majority of $\mathrm{ADHs},{ }^{[19]}$ a more variable (often aromatic) bulky moiety as the large one. ${ }^{[20]}$ The preferred LBADH substrate for in vitro studies is acetophenone (Figure 1A), which is reduced to enantiopure $(R)-1$ - phenylethanol (Figure 1B). The stereoselectivity results from an active site that offers two hydrophobic pockets: a small flexible one and a larger open area, which host the small and large substituents of the substrate, respectively. In order to analyze any possible contribution of substrate-enzyme interactions to the degrees of conversion listed in Table 1, we compared the binding modes obtained for the reference ketone (2b) and its corresponding alcohol $[(R)-\mathbf{1 b}]$ to those of $(S)-\mathbf{1 c} / \mathbf{2 c}$ (see Experimental Section and Supporting Information for details), which is the pair with the largest difference in conversion and structurally most similar to the reference substrates.

For the case of ketone 2c (Figure 1C) the active site of LBADH remained in the same conformation as in the complex with acetophenone (2b, Figure 1A), thus indicating that the active site has enough space to accommodate the halogen atom. Thus, the $\mathrm{C}-\mathrm{Cl}$ bond is oriented in an antiparallel fashion with respect to the plane of the phenolic ring of Tyr189, amino acid that lies at the entrance of the small pocket of the active site of LBADH. In turn, the binding mode obtained for $(S)$-2-chloro-1-phenylethanol [ $(S)$-1c, Figure 1D] was qualitatively identical to that of 2-chloroacetophenone (2c, Figure 1C). However, a slightly different interaction of the chlorine atom with the aryl ring of Tyr189 (Figure 1D) is observed. Thus, the $\mathrm{C}-\mathrm{Cl}$ bond is perpendicular to the plane of the phenolic ring of this amino acid, thus resembling the face-to-edge interaction mode of aryl rings. Apart from this, the binding modes for the $\mathbf{1 c} / \mathbf{2 c}$ pair are identical to those obtained for the $\mathbf{1 b} / \mathbf{2 b}$ one. These results confirm that there is no structural reason that would block the oxidation of $(S)-\mathbf{1 c}$ but not the one of alcohol $\mathbf{1 b}$ and, at the same time, would allow the reduction of ketone 2c. Consequently, if this ketone is reduced, its alcohol should be equally oxidized.

\section{Metal-catalyzed reduction of ketones under HT conditions}

The lack of enzyme-substrate interactions that could block the enzymatic oxidation of the halohydrin (S)-1c suggests that conversions listed in Table 1 are not due to the properties of the enzyme but to the nature of the substrates. In order to confirm this hypothesis we used a different catalyst. In particular, the MPVO-HT of ketones $\mathbf{2} \mathbf{b}$-h was carried out (Table 2) using $\mathrm{Al}\left({ }^{t} \mathrm{BuO}\right)_{3}$ in situ activated with trifluoroacetic acid as catalyst, ${ }^{[21]}$ and 2 equiv. of 2 propanol. Methyl 4-chloroacetoacetate (2h) was the only substrate that could not be reduced under these conditions because it decomposed. We have attributed this behavior to side aldol 
condensations, which are known to be potentiated by mixtures of aluminum alkoxides and protic acids, especially when easily enolizable ketones like $\mathbf{2 h}$ are used. ${ }^{[4 b, c]}$ Apart from this substrate, the results obtained were indeed comparable to those afforded by LBADH (Table 1): higher conversions were observed for those ketones with electron-withdrawing groups and the negligible differences between the results of the enzymatic and the metalcatalyzed reactions can be attributed to the different reaction conditions employed.

\begin{tabular}{lllll}
\hline Entry & Compound & $\mathrm{R}^{1}$ & $\mathrm{R}^{2}$ & conv [\%] \\
\hline 1 & $\mathbf{2 b}$ & $\mathrm{Ph}$ & $\mathrm{Me}$ & 52 \\
2 & $\mathbf{2 c}$ & $\mathrm{Ph}$ & $\mathrm{CH}_{2} \mathrm{Cl}$ & 95 \\
3 & $\mathbf{2 d}$ & $\mathrm{Ph}$ & $\mathrm{CH}_{2} \mathrm{~N}_{3}$ & 84 \\
4 & $\mathbf{2 e}$ & $p-\mathrm{O}_{2} \mathrm{~N}-\mathrm{Ph}$ & $\mathrm{Me}$ & 78 \\
5 & $\mathbf{2 f}$ & $p-\mathrm{MeO}^{-\mathrm{Ph}_{2}}$ & $\mathrm{Me}$ & 12 \\
6 & & & & 79 \\
7 & $\mathbf{2 g}$ & $\mathrm{MeOCH}_{2}$ & $\mathrm{Me}$ & n.a. \\
\hline
\end{tabular}

Table 2. Meerwein-Ponndorf-Verley reduction of ketones $\mathbf{2} \mathbf{b}-\mathbf{h}$ catalyzed by $\mathrm{Al}\left({ }^{t} \mathrm{BuO}\right)$ employing 2 equiv. of 2-propanol.

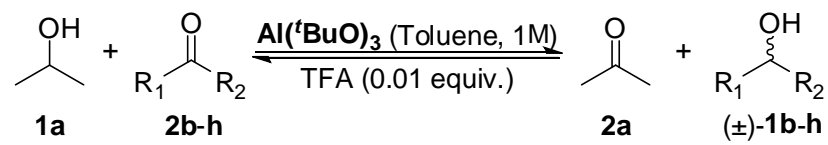

2 equiv. 1 equiv.

n.a. not available.
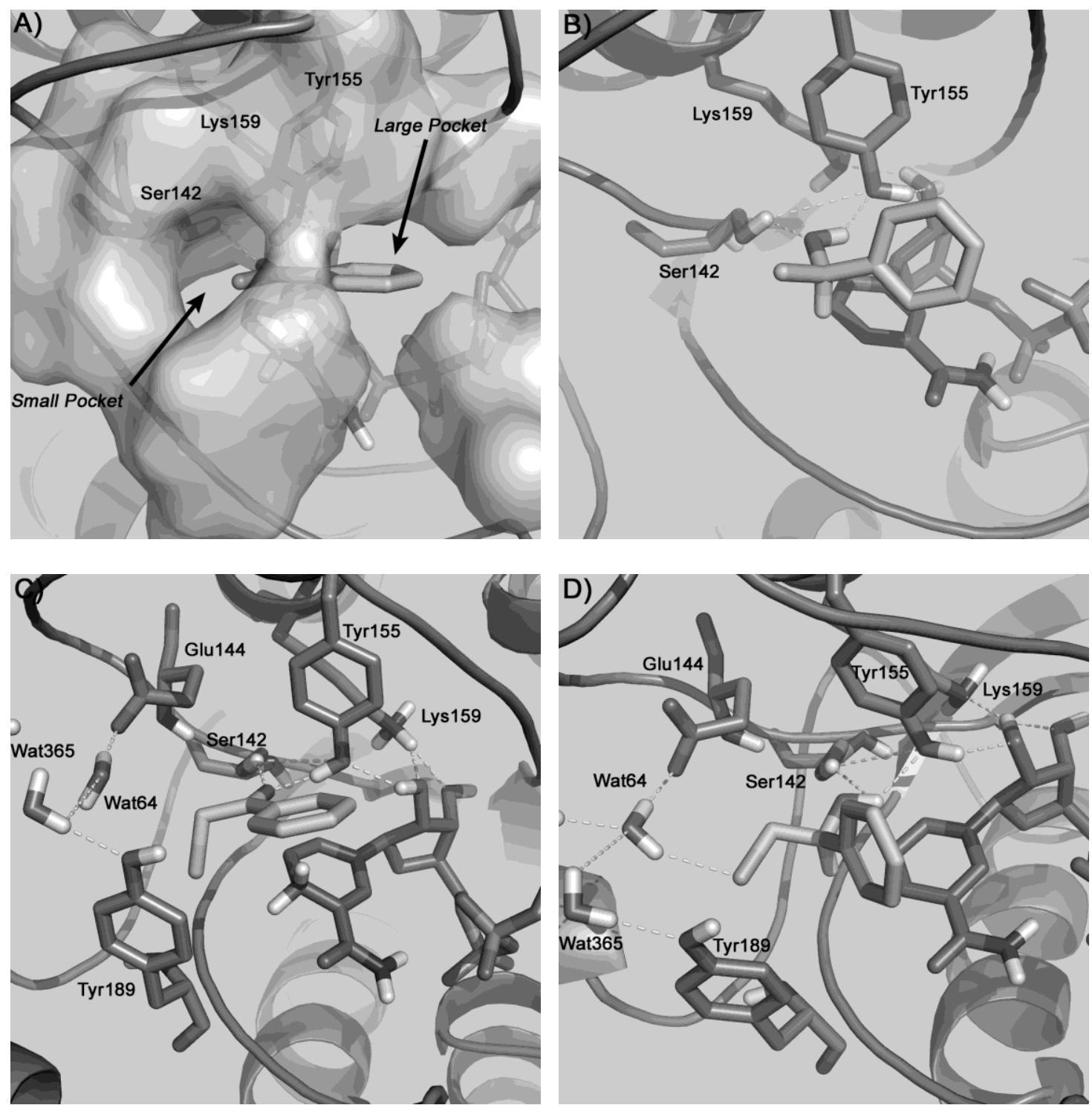

Figure 1. Modeled complexes of LBADH with: A) acetophenone (2b), B) $(R)$-1-phenylethanol $[(R)$-1b], and C) 2-chloroacetophenone (2c) and $(S)$-2-chloro-1-phenylethanol [(S)1c]. Only polar and reactive hydrogens are shown. The carbon atoms of the ligand, cofactor, and amino acids are shown in cyan, magenta, and slate, respectively. Key amino acids are labeled. 


\section{Initial rates of the LBADH-catalyzed HT of ketones $2 b-h$}

At this point, we were intrigued by the question of why ketones bearing EWGs oxidized ${ }^{i} \mathrm{PrOH}$ more efficiently. As it has already been mentioned, this behavior has been associated to a higher electrophilicithy of the carbonyl carbon, ${ }^{[16]}$ which should be reflected in the kinetics of the reaction. Accordingly, we measured the initial velocities $\left(V_{0}\right)$ of the LBADH-catalyzed reduction of ketones 2c-h (see Supporting Information for details) and calculated their ratio with respect to the parent substrate (2b; Figure 2$)$. As a general trend, ketones reduced with higher degrees of conversion showed indeed higher initial rates. Thus, 2-chloroacetophenone (2c) or $p$-nitroacetophenone (2e) were reduced $c a 5$-times faster than the reference substrate $\mathbf{2 b}$. Also, when the ketone bears two electronwithdrawing groups $(\mathbf{2} \mathbf{h})$, the enhanced effect on $V_{0}$ was even more pronounced. On the contrary, ketone 2f, which showed lower conversion, also presented a smaller initial rate. However, this correlation was not always true. Thus, the reduction of 2azidoacetophenone (2d) was approx. 20-fold slower than that of the parent substrate (2b), but it proceeded almost quantitatively. Although the lower $V_{0}$ of this ketone could be attributed to the larger size of the azido group as compared to the reference substrate, this is not the case of compound $\mathbf{2 g}$. This ketone should perfectly fit in the active site of the enzyme, and despite presenting an almost identical $V_{0}$ to that of the reference compound, it led to a much higher degree of conversion. Therefore, these data allowed us to draw the following conclusions: 1) ketones with EWGs are, in general, more readily reduced than electron-rich ones; but 2) no general correlation between initial velocities and degree of conversions can be established, thus ruling out any dominant kinetic contribution to the conversions shown in Table 1. Consequently, the reactions should be, at least mainly, under thermodynamic control.

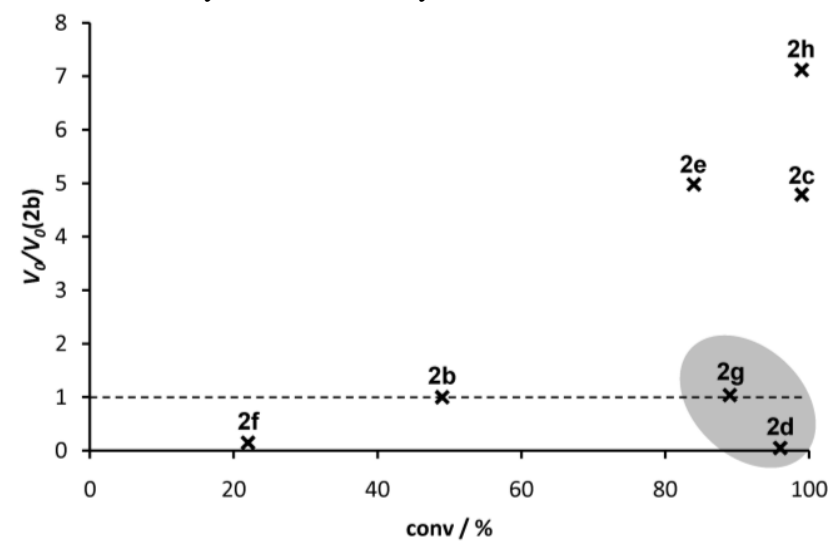

Figure 2. Ratio of the initial rates of the LBADH-catalyzed reduction of ketones $\mathbf{2 c}-\mathbf{h}$ with respect to that of ketone $\mathbf{2 b}$ plotted against the degrees of conversion. Outliers are highlighted in grey.

\section{IR carbonyl stretching bands}

Neuvonen and Neuvonen have studied the kinetics of the nucleophilic acyl substitutions of different esters and found relationships between different spectroscopic parameters of the carbonyl group and the rates observed for a series of structurally related compounds. ${ }^{[22]}$ They observed that the IR absorption frequencies of the ester carbonyl group unexpectedly increased with the reaction rate. Therefore, esters bearing EWGs showed higher frequencies (higher double bond character), which contradicted the tacit assumption of increased electrophilicity of the carbonyl carbon. Alternatively, the authors attributed this rate acceleration to the energetics of the ester substrate: EWGs would decrease the contribution of the dipolar ester resonance form and hence, would destabilize the ground state resonance of the substrate. Inspired by this work and taking into account that a keto function is also constituted by a mixture of two resonance forms (Figure 3), we measured the frequencies of the IR carbonyl absorption bands of ketones 2b-h (see Supporting Information, Table S4) and plotted them against the degrees of conversion at equilibrium shown in Tables 1 and 2 (Figure 3). ${ }^{[23]}$ As can be seen, the presence of EWGs also leads to higher frequencies, ${ }^{[24]}$ and a good correlation between them and the conversions was observed: higher values of the carbonyl stretching band (right zone) correspond to ketones reduced with higher degrees of conversion and vice versa (left zone). Similarly to the case of esters, this tendency can also be attributed to the fact that ketones with EWGs should be more unstable because the dipolar form is now disfavored, which destabilizes their ground resonance form. If the transition state is not affected by the substitution, this would make them more reactive but, more importantly, should affect the energy difference of the pairs alcohol/ketone and hence the equilibrium constant. This hypothesis goes in hand with a thermodynamic control of the HT reactions and shows that a preliminary IR spectrum can be safely used as a reliable method to qualitatively predict if a ketone will be a good hydrogen acceptor under HT conditions even for structurally different ketones. ${ }^{[23]}$
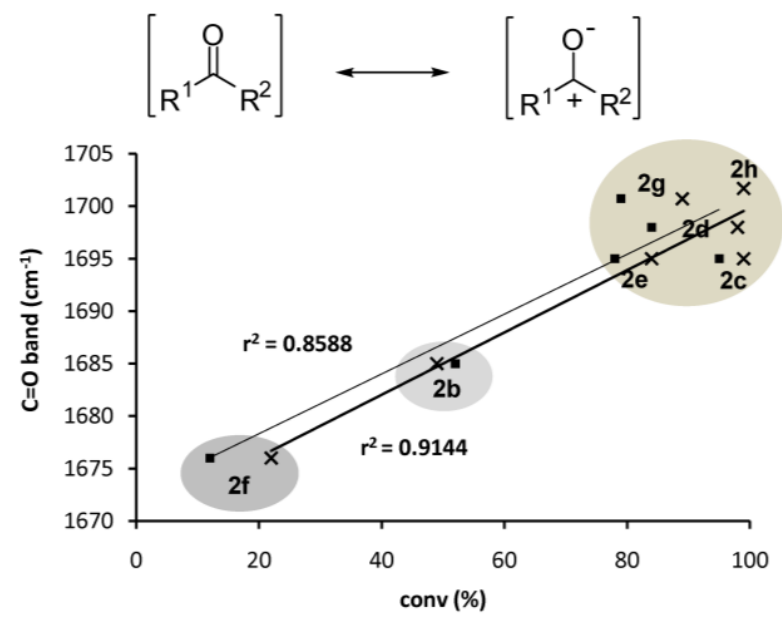

Figure 3. Ketone resonance structures and plot of IR carbonyl stretching bands of substrates 2b-h against the degrees of conversion for: LBADH- (crosses) and aluminum-catalyzed (squares) HT reactions.

\section{$A b$ initio calculations}

The results obtained so far indicate that neither the catalyst nor the kinetics are responsible for the different conversions observed in the HT transformations, and suggest that this behavior has to be mainly attributed to the thermodynamics of the reaction (i.e., the $\Delta E^{O}$ differences between the 2-propanol/acetone and the alcohol/ketone counterpart). To corroborate this, the Gibbs free energies for the isodesmic transformations shown in Table 3 have been calculated in both solution and gas phases. 
Table 3. Total number of conformers $\left(N_{\text {conf }}\right)$ of the alcohol/ketone pairs examined computationally and Boltzmann-averaged free energies $\left(\mathrm{kcal} \mathrm{mol}^{-1}\right)$ both in solution $\left(\Delta \bar{G}_{a q}\right)$ and in the gas-phase ( $\Delta \bar{G}_{\text {gas-phase }}$ ) for the redox equilibrium with respect to 2propanol/acetone. The corresponding changes in the free energy as evaluated over the most stable conformers in solution ( $\Delta G_{a q}^{\min }$ ) are also indicated.

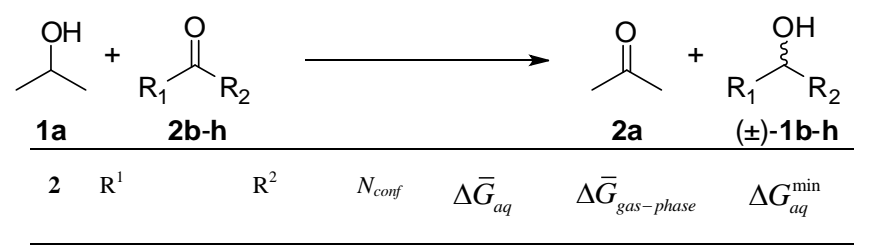

\begin{tabular}{|c|c|c|c|c|c|c|}
\hline $\mathbf{a}$ & $\mathrm{Me}$ & $\mathrm{Me}$ & $1 / 1$ & - & - & - \\
\hline b & $\mathrm{Ph}$ & $\mathrm{Me}$ & $2 / 1$ & -0.61 & -0.20 & -0.36 \\
\hline c & $\mathrm{Ph}$ & $\mathrm{CH}_{2} \mathrm{Cl}$ & $6 / 2$ & -4.86 & -4.98 & -4.29 \\
\hline d & $\mathrm{Ph}$ & $\mathrm{CH}_{2} \mathrm{~N}_{3}$ & $12 / 2$ & -5.10 & -4.86 & -4.17 \\
\hline e & $p-\mathrm{O}_{2} \mathrm{~N}-\mathrm{Ph}$ & $\mathrm{Me}$ & $3 / 1$ & -1.65 & -1.16 & -0.92 \\
\hline $\mathbf{f}$ & $p$-MeO-Ph & $\mathrm{Me}$ & $4 / 2$ & 0.56 & 0.71 & 1.17 \\
\hline $\mathbf{g}$ & $\mathrm{MeOCH}_{2}$ & $\mathrm{Me}$ & $16 / 3$ & -3.55 & -3.85 & -3.07 \\
\hline h & $\mathrm{MeO}_{2} \mathrm{CCH}_{2}$ & $\mathrm{CH}_{2} \mathrm{Cl}$ & $57 / 9$ & -7.04 & -7.15 & -5.82 \\
\hline
\end{tabular}

Analysis of the $\Delta \bar{G}_{a q}$ values showed that quantum chemical results agreed quite well with the experimental data and consequently the same trends were observed. In other words, reactions where ketones, either aromatic or aliphatic, contain electronegative groups in $\alpha$-position $(\mathbf{2 c}, \mathbf{2 d}, \mathbf{2 g}$, and $\mathbf{2 h}$ ) had lower $\Delta \bar{G}_{a q}$ than ketones containing deactivating substituents in the aromatic ring (2e). Acetophenone (2b) showed a value close to 0 $\mathrm{kcal} \cdot \mathrm{mol}^{-1}$, and expectedly, the reduction of ketone $\mathbf{2 f}$ was disfavored. Solvation effects were clearly not determining since differences between $\Delta \bar{G}_{a q}$ and $\Delta \bar{G}_{\text {gas-phase }}$ were systematically below $0.5 \mathrm{kcal} \mathrm{mol}^{-1}$ and the exact same trends were predicted in both environments.

The influence of the conformational flexibility of each reactant and product were also examined by comparing $\Delta \bar{G}_{a q}$ and $\Delta G_{a q}^{\mathrm{min}}$, where the former was obtained as an average over the most stable conformers of each particular structure according to a MaxwellBoltzmann distribution and the latter was calculated considering only the most stable conformer for each substrate. Even when $\Delta G_{a q}^{\text {min }}$ energies were slightly higher than $\Delta \bar{G}_{a q}$, the information obtained from both sets of energies was practically equivalent. From this assessment it may seem that performing a conformational analysis was irrelevant for the calculation of free energies of reaction. However, although restricting the calculation of the Gibbs free energies of reaction to the most stable conformer had no significant effect in $\Delta \bar{G}_{a q}$ from a quantitative point of view, in terms of finding true reasons explaining the shift of the redox equilibrium, skipping the conformational complexity of a certain system may lead to misleading conclusions. To illustrate this point, we have focused on substrate 1c. In principle, it would not be misconceived to think that oxidation of 2-chloro-1-phenylethanol to 2-chloroacetophenone can be hampered by the fact that for the former an $\mathrm{OH} \cdots \mathrm{Cl}$ intramolecular $\mathrm{H}$-bond can be formed. ${ }^{[11]}$ Such intramolecular H-bond occurred in two of the six existing conformers in the conformational landscape, specifically in the most stable conformer and in the fourth local minima (according to Gibbs free energies in solution). However, it was not present in the four remaining conformers, which were very close in energy to the previous ones (all together the six conformers lay within an interval of free energy of $1 \mathrm{kcal} \mathrm{mol}^{-1}$ ). In other words, the co-existence of the six conformers in solution is incompatible with the shift of the redox reaction towards 2-chloro-1-phenylethanol due to a formation of an intramolecular H-bond in the latter one. This is in agreement with the fact that for 2-azidoacetophenone (where such an intramolecular H-bond is not possible), the degree of conversion as well as the $\Delta \bar{G}_{a q}$ value were very similar to those obtained for 2 chloroacetophenone. Therefore we conclude that none of the above mentioned factors (solvation effects and conformational flexibility) have a large impact in the Gibbs free energies of reaction meaning that the constant of the redox equilibrium is mostly depending on the intrinsic stability of the alcohol/ketone pairs considered.

Taking into account the good correlation obtained between the IR absorption bands of the carbonyl group and the experimental conversions, we examined several theoretical descriptors of the reactive $\mathrm{C}=\mathrm{O}$ bond in order to confirm the hypothesis about the resonance (de)stabilization of ketones upon substitution (Table S6 in the Supporting Information). Thus, either the bond distance of the $\mathrm{C}=\mathrm{O}$ bond (Figure $\mathrm{S} 2 \mathrm{~A}$ ) or its charge density at the corresponding Bond Critical Point (BCP, Figure S2B) correlate reasonably well with the average free energy in solution $\left(\Delta G_{\mathrm{aq}}\right)$ for the reduction of 2b-g by 2-propanol: the shorter the $\mathrm{C}=\mathrm{O}$ bond distance (or the larger accumulation of charge density at the $\mathrm{BCP}$ ) was, the more favorable the reduction of the keto groups was. We also examined the variation in the local energy density at the $\mathrm{C}=\mathrm{O} \mathrm{BCPs}\left[H\left(\mathrm{r}_{\mathrm{c}}\right)\right]$ because this quantity is a stable property with regard to the $a b$ initio level of theory (Figure $\mathrm{S} 2 \mathrm{C}$ ). For all the $\mathrm{C}=\mathrm{O}$ bonds, the sign of $H\left(r_{c}\right)$ is negative, what indicates a predominant covalent nature. $H\left(\mathrm{r}_{\mathrm{c}}\right)$ showed a good correlation with the relative free energies, showing thus that the more ionic or polarized is the $\mathrm{C}=\mathrm{O}$ bond [i.e., the lower $H\left(\mathrm{r}_{\mathrm{c}}\right)$ in absolute value], the less likely is to be reduced. This observation reinforces the hypothesis of the destabilization of the ketones based on the experimental IR absorption bands (Figure $3)$.

\section{Overcoming HT limitations combining halohydrins with $\alpha$ - haloketones}

At this point, we were interested in oxidizing alcohols thermodynamically impeded (i.e. halohydrins) under standard HT conditions. As the combination of an activated ketone (e.g., 2chloroacetophenone) with a non-activated alcohol (e.g., 2-propanol) led to complete oxidation of the alcohol due to the high $\Delta E^{0}$ difference between both pairs, employing a pair of substrates both containing activated ketones, should lead to an equilibrium far away from quasi-quantitative conversions. In order to prove this, we performed the reduction of ketone $\mathbf{2 c}$ using 1-chloro-2-propanol (1i) as hydrogen acceptor, ${ }^{[25]}$ and the opposite transformation (alcohol 1c with chloroacetone 2i) employing both $\mathrm{LBADH}$ and $\mathrm{Al}\left({ }^{t} \mathrm{BuO}\right)_{3}$ (Scheme 2). 


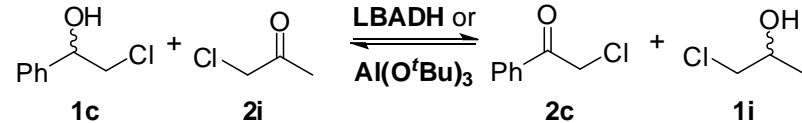

Scheme 2. Reduction of $\alpha$-haloketones in HT conditions using halohydrins as hydrogen acceptors.

By using LBADH and different ratios of the hydrogen acceptor and donor (from 1:2 until 4:1), we observed enzymatic conversions in the range of $3-12 \%$ in both directions (starting materials $\mathbf{1 c}+\mathbf{2 i}$ or 2c+1i) after $24 \mathrm{~h}$. Leaving these reactions longer ( 7 days) and adding fresh biocatalyst and cofactor every day, ${ }^{[26]}$ led to higher conversions $(>30 \%)$. When the aluminum catalyst was employed with 2 equiv. of the halohydrin and 1 equiv. of the $\alpha$-chloroketone, conversions around $20 \%$ were achieved after $24 \mathrm{~h}$. Therefore, these results clearly demonstrate, on the one hand, that the oxidation of halohydrins is not blocked and that they can be oxidized under HT conditions by selecting an acceptor with a suitable redox potential. On the other hand, the different concentrations of products measured for the forward and reverse reactions clearly show that the thermodynamic equilibrium was not reached after $24 \mathrm{~h}$. Therefore, the oxidation of halohydrins is also under some kinetic control, as it is further supported by the fact that the higher temperature of the metal-catalyzed HT reaction as compared to the enzymatic one, allowed higher conversions.

\section{Conclusions}

We have studied the redox equilibrium of a series of ketones in order to understand the extent of their HT reduction. As a general feature, higher enzymatic conversions are obtained with substrates bearing EWGs regardless the catalyst employed. This suggests that the observed conversions are due to the intrinsic nature of the substrates. In this same sense, the docking of $\alpha$-haloketone $2 \mathbf{c}$ and its corresponding alcohol (1c) in the active site of LBADH suggests that the quasi-quantitative conversion attained is not likely due to any enzyme-substrate interaction that could block the oxidation of the halohydrin.

The higher reactivity of ketones bearing EWGs has been traditionally attributed to a higher electrophilicity of the carbonyl carbon because of the inductive effect of these substituents. However, the initial rates of reaction do not always correlate with the conversions, and the normalized IR frequencies of the carbonyl groups not only suggest the opposite but show a good correlation with the experimental conversions, thus affording a straightforward and easy predictive method of the position of the equilibrium that will be attained for a given ketone. Therefore, it seems that the extent of the reduction of these ketones is not under kinetic control and, hence, it should be under thermodynamic one. This is indeed in agreement with the good correlation observed for the conversions and the calculated Gibbs free energy ketone/alcohol differences against 2-propanol/acetone. This behavior has been explained on the basis that the presence of EWGs would destabilize the ground resonance state of the ketones by decreasing the contribution of the dipolar resonant form. This hypothesis explains the general higher reactivity of these substrates and suggests that the equilibrium constants of these reactions are mainly driven by the different energies of the ketones. Moreover, the higher contribution of the double-bond resonant form leads to higher frequencies in the absorption band, which was further confirmed by the calculated values of different suitable descriptors of the $\mathrm{C}=\mathrm{O}$ bond.

Once the lack of reactivity of halohydrins was clarified, it became evident that the oxidation of halohydrins and related alcohols should be feasible by using, as hydrogen acceptor, another ketone bearing an EWG. Such reactions did indeed proceed to a relatively high extent but they did not reach the equilibrium in the time frames used. Therefore, for the case of alcohols bearing EWGs, the oxidation is slowed down as compared to other hydrogen donors, and longer reaction times or higher temperatures are required to reach the equilibrium concentrations.

\section{Experimental Section}

General experimental methods. Alcohol dehydrogenases and ketones 1a-i were purchased from commercial sources. Racemic alcohols $( \pm)$-2a-i were either synthesized by conventional reduction of the corresponding ketones $\left(\mathrm{NaBH}_{4}, \mathrm{MeOH}\right.$, r.t.) or purchased from commercial sources. All other reagents, including catalysts and solvents were of the highest quality available. Flash chromatography was performed using silica gel 60 (230-400 mesh). ${ }^{1} \mathrm{H}-,{ }^{13} \mathrm{C}-\mathrm{NMR}$, and DEPT were obtained using DPX-300 $\left({ }^{1} \mathrm{H}\right.$, $300.13 \mathrm{MHz}$ and ${ }^{13} \mathrm{C}, 75.5 \mathrm{MHz}$ ) spectrometer for routine experiments. Gas chromatography (GC) analyses were performed on a standard gas chromatograph using nitrogen as carrier. UV and IR spectra were performed on standard UV/vis and IR spectrophotometers, respectively.

Enzymatic hydrogen transfer protocol using LBADH. Generally, in an Eppendorf vial $(1.5 \mathrm{~mL}), \mathrm{LBADH}(3 \mathrm{U})$ was added into $600 \mu \mathrm{L}$ of Tris- $\mathrm{HCl}$ buffer $(50 \mathrm{mM}, \mathrm{pH} 7.5$, $\left.1 \mathrm{mM} \mathrm{MgCl}_{2}, 1 \mathrm{mM} \mathrm{NADPH}\right)$. Then, the corresponding ketone (25 mM) and 2-propanol $\left(50 \mathrm{mM}, 1.8 \mu \mathrm{L}, 2\right.$ equiv.) were added to the mixture. Reactions were shaken at $30^{\circ} \mathrm{C}$ and $150 \mathrm{rpm}$ for $24 \mathrm{~h}$. Then, reactions were stopped by extraction with ethyl acetate $(2 \mathrm{x}$ $0.6 \mathrm{~mL})$. The organic layer was separated by centrifugation $(1.5 \mathrm{~min}, 13000 \mathrm{rpm})$ and dried over $\mathrm{Na}_{2} \mathrm{SO}_{4}$. Conversions were determined by GC analysis.

Hydrogen transfer protocol using $\mathrm{Al}\left({ }^{t} \mathrm{BuO}\right)_{3}$. In a sealed Schlenk tube, $\mathrm{Al}\left({ }^{t} \mathrm{BuO}\right)_{3}$ $(100 \mu \mathrm{L}$ of a $0.1 \mathrm{M}$ solution in dry toluene), the corresponding ketone $(0.5 \mathrm{mmol}), 2$ propanol $(1 \mathrm{mmol}, 77 \mu \mathrm{L})$ and trifluoroacetic acid $(100 \mu \mathrm{L}$ of a $0.1 \mathrm{M}$ solution in dry toluene) were added under $\mathrm{N}_{2}$ atmosphere to $4 \mathrm{~mL}$ of dry $\mathrm{CH}_{2} \mathrm{Cl}_{2}$. Then, the sealed tube was stirred at $60{ }^{\circ} \mathrm{C}$ for $24 \mathrm{~h}$. To determine conversions, $500 \mu \mathrm{L}$ aliquots were withdrawn, the catalyst was quenched with one drop of water and $\mathrm{Na}_{2} \mathrm{SO}_{4}$ was added. Conversions were determined by GC analysis.

General protein-ligand computational methods. All molecular mechanics computations were performed with the molecular modeling package Molecular Operating Environment (MOE) 2007.09 (Chemical Computing Group, Inc.). ${ }^{[27]}$ In all cases the Amber99 force field, ${ }^{[28]}$ the corresponding dictionary charges as implemented in MOE, and the Generalized Born solvation model ${ }^{[29]}$ with relative dielectric constant values of 2 and 80 for the protein matrix ${ }^{[30]}$ and the solvent, respectively, were selected. A non-bonded cut-off of $8 \AA$ with a smoothing function between 8 and $10 \AA$ were used. In all Molecular Dynamics Simulations (MDS) the NVT ensemble and the NoséPoincaré-Anderson equations were selected. The initial and simulation temperatures were set to 0 and $300 \mathrm{~K}$, respectively, a temperature relaxation time of $10 \mathrm{fs}$ was selected, and the length of the heating and simulation periods was $1 \mathrm{ps}$. No constraints were imposed on any bond and a step size of $1 \mathrm{fs}$ was used. The convergence criterion of the Energy Minimizations (EM) was set to a gradient value of $0.01 \mathrm{kcal} \mathrm{mol}^{-1}$ or $0.001 \mathrm{kcal} \mathrm{mol}^{-1}$ when only parts of the protein or the whole enzyme-substrate-cofactor complexes were minimized, respectively. The rest of parameters were set to their default values. High quality pictures of representative structures were generated with PyMOL 0.99. ${ }^{[31]}$

$\boldsymbol{A} \boldsymbol{b}$ initio calculations. Gibbs free energies of reactants and products corresponding to the reaction described in Table 1 were calculated according to the following equation:

$$
\bar{G}_{a q}=\bar{G}_{\text {thermal }}^{H F}+\bar{E}_{M P 2 / C B S}+\Delta \bar{G}_{\text {solv }}^{\text {COSMO }}-T S_{\text {conf }}
$$

where $G_{\text {thermal }}^{H F}$ stands for the thermal contributions (translational, rotational and vibrational) to Gibbs free energy, $E_{\mathrm{MP} 2 / \mathrm{CBS}}$ is the MP2 electronic energy evaluated at the complete basis set (CBS) limit, $\Delta G_{\text {solv }}^{\operatorname{COSMO}}$ is the solvation free energy and $S_{\text {conf }}$ is the conformational entropy. In this expression, the energy components (except $S_{\text {conf }}$ ) are averaged over all the existing conformers of each particular structure according to a Maxwell-Boltzmann distribution. 
For the isodesmic transformations, energies were averaged over the most stable conformers $\left(N_{c o n f}\right)$ of each particular structure according to a Maxwell-Boltzman distribution. For the sake of completeness, relative free energies of reaction have been calculated in solution ( $\Delta \bar{G}_{a q}$ ) as well as in the gas-phase ( $\Delta \bar{G}_{\text {gas-phase }}$ ). Relative free energies ( $\Delta G_{a q}^{\mathrm{min}}$ ) evaluated over the most stable conformers in solution are also given

\section{Acknowledgements}

F.R.B. is supported by the Programme Alßan, the European Union Program of High Level Scholarships for Latin America (scholarship No. E07D402519AR). I.L. thank Principado de Asturias for personal funding (Clarín Program). Financial support from the Spanish Ministerio de Ciencia e Innovación (MICINN, Project CTQ2007 61126/PPQ) is gratefully acknowledged. Prof. F. López Ortiz is gratefully acknowledged for helpful discussions.

[1] M. A. Johnson, Nature Chem. 2009, 1, 8-9.

[2] The Principles of Chemical Equilibrium, (Ed.: K. G. Denbigh), Cambridge University Press, Cambridge, 1981.

[3] (a) X. Wu, J. Xiao, Chem. Commun. 2007, 2449-2466; (b) T. Ikariya, A. J. Blacker, Acc. Chem. Res. 2007, 40, 1300-1308; (c) S. Gladiali, E. Alberico, Chem. Soc. Rev. 2006, 35, 226-236; (d) T. Ikariya, K. Murata, R. Noyori, Org Biomol. Chem. 2006, 4, 393-406; (e) D. Lenoir, Angew. Chem. 2006, 118, 32803284; Angew. Chem. Int. Ed. 2006, 45, 3206-3210; (f) M. J. Schultz, M. S. Sigman, Tetrahedron 2006, 62, 8227-8241; (g) Modern Oxidation Methods, (Ed.: J.-E. Bäckvall), Wiley-VCH, Weinheim, 2004.

[4] For some reviews, see: (a) G. K. Chuah, S. Jaenicke, Y. Z. Zhu, S. H. Liu, Curr Org. Chem. 2006, 10, 1639-1654; (b) C. R. Graves, E. J. Campbell, S. T Nguyen, Tetrahedron: Asymmetry 2005, 16, 3460-3468; (c) R. Noyori, M Yamakawa, S. Hashiguchi, J. Org. Chem. 2001, 66, 7931-7944; (d) C. F. de Graauw, J. A. Peters, H. van Bekkum, J. Huskens, Synthesis 1994, 1007-1017.

[5] Some recent bibliography: (a) K. Nakamura, T. Matsuda in Asymmetric Organic Synthesis with Enzymes, (Eds.: V. Gotor, I. Alfonso, E. García-Urdiales), WileyVCH, Weinheim, 2008, pp. 193-228; (b) S. Buchholz, H. Gröger in Biocatalysis in the Pharmaceutical and Biotechnology Industry, (Ed.: R. N. Patel), CRC Press, Boca Raton, 2007, pp. 757-790; (c) S. M. A. de Wildeman, T. Sonke, H. E. Schoemaker, O. May, Acc. Chem. Res. 2007, 40, 1260-1266; (d) J. C. Moore D. J. Pollard, B. Kosjek, P. N. Devine, Acc. Chem. Res. 2007, 40, 1412-1419, (e) K. Goldberg, K. Schroer, S. Lütz, A. Liese, Appl. Microbiol. Biotechnol. 2007, 76, 237-248; (f) E. García-Urdiales, I. Alfonso, V. Gotor, Chem. Rev. 2005, 105, 313-354; (g) W. Kroutil, H. Mang, K. Edegger, K. Faber, Curr. Opin. Chem. Biol. 2004, 8, 120-126.

[6] (a) R. A. Sheldon, Pure Appl. Chem. 2000, 72, 1233-1246. (b) B. M. Trost Science 1991, 254, 1471-1477.

[7] There have been described protocols in order to shift the equilibrium in ADH catalyzed HT transformations by means of medium engineering employing ionic liquids: M. Eckstein, M. V. Filho, A. Liese, U. Kragl, Chem. Commun. 2004, 1084-1085; or by continuous removing of acetone from the reaction medium resembling an in situ product recovery (ISPR) example: T. Stillger, M. Bönitz, M. V. Filho, A. Liese, Chem. Ing. Tech. 2002, 74, 1035-1039.

[8] F. R. Bisogno, I. Lavandera, W. Kroutil, V. Gotor, J. Org. Chem. 2009, 74, 1730-1732.

[9] I. Lavandera, A. Kern, V. Resch, B. Ferreira-Silva, A. Glieder, W. M. F. Fabian, S. de Wildeman, W. Kroutil, Org. Lett. 2008, 10, 2155-2158.

[10] (a) M. F. Eckstein, M. Peters, J. Lembrecht, A. C. Spiess, L. Greiner, Adv. Synth Catal. 2006, 348, 1591-1596; (b) M. F. Eckstein, J. Lembrecht, J. Schumacher, W. Eberhard, A. C. Spiess, M. Peters, C. Roosen, L. Greiner, W. Leitner, U. Kragl, Adv. Synth. Catal. 2006, 348, 1597-1604.

[11] T. Goldstein, M. S. Snow, B. J. Howard, J. Mol. Spectrosc. 2006, 236, 1-10.
[12] K. Krohn, B. Knauer, J. Küpke, D. Seebach, A. K. Beck, M. Hayakawa, Synthesis 1996, 1341-1344.

[13] (a) E. J. Campbell, H. Zhou, S. T. Nguyen, Angew. Chem. 2002, 114, 10621064; Angew. Chem. Int. Ed. 2002, 41, 1020-1022; (b) E. J. Campbell, H. Zhou, S. T. Nguyen, Org. Lett. 2001, 3, 2391-2393.

[14] R. Mello, J. Martínez-Ferrer, G. Asensio, M. E. González-Núñez, J. Org. Chem 2007, 72, 9376-9378.

[15] For other quasi-irreversible bio- or metal-catalyzed HT transformations employing other type of reagents, see: (a) M. Kurina-Sanz, F. R. Bisogno, I. Lavandera, A. A. Orden, V. Gotor, Adv. Synth. Catal. 2009, 351, 1842-1848; (b) C. Peschko, J. Stohrer, Wacker Chemie AG, Germany, DE 102006009743, A1 20070906, CAN 2007, 147:321414; (c) H. C. Maytum, B. Tavassoli, J. M. J. Williams, Org. Lett. 2007, 9, 4387-4389; (d) N. J. Wise, J. M. J. Williams, Tetrahedron Lett. 2007, 48, 3639-3641.

[16] N. P. Gambaryan, E. M. Rokhlin, Y. V. Zeifman, C. Ching-Yung, I. L. Knunyants, Angew. Chem. 1966, 78, 1008-1017; Angew. Chem. Int. Ed. 1966, 5 , 947-956.

[17] M. Wolberg, W. Hummel, C. Wandrey, M. Müller, Angew. Chem. 2000, 112, 4476-4478; Angew. Chem. Int. Ed., 2000, 39, 4306-4308.

[18] To ensure that these results were not only obtained with this short-chain ADH due to any especial feature, we have repeated these experiments with the NADH medium-chain alcohol dehydrogenase from Rhodococcus ruber ADH-'A', showing a very similar trend. For more information, see Reference 8.

[19] W. Hummel, Adv. Biochem. Eng. Biotechnol. 1997, 58, 145-184.

[20] N. H. Schlieben, K. Niefind, J. Muller, B. Riebel, W. Hummel, D. Schomburg, $J$. Mol. Biol. 2005, 349, 801-813.

[21] K. G. Akamanchi, V. R. Noorani, Tetrahedron Lett. 1995, 36, 5085-5088.

[22] H. Neuvonen, K. Neuvonen, J. Chem. Soc., Perkin Trans. 2 1999, 1497-1502.

[23] To normalize the carbonyl IR frequencies so that structurally different ketones can be reliably compared, a correction factor of 0.982 was applied to the IR frequencies of aliphatic substrates $\mathbf{2 g}$ and $\mathbf{2 h}$. This factor comes from the division of the carbonyl stretching frequencies of acetophenone $\left(1685 \mathrm{~cm}^{-1}\right)$ and acetone $\left(1715 \mathrm{~cm}^{-1}\right)$. By doing so, we have assumed that IR frequencies are only dependant on the strength of the carbonyl bond and the size of its substituents.

[24] R. N. Jones, E. Spinner, Can. J. Chem. 1958, 36, 1020-1027.

[25] We have chosen $\mathbf{1 i} / \mathbf{2} \mathbf{i}$ due to the problems observed with substrate $\mathbf{2} \mathbf{h}$ in the HT conditions catalyzed by $\mathrm{Al}\left(\mathrm{O}^{t} \mathrm{Bu}\right)_{3}$. We have also performed the enzymatic reactions coupling $1 \mathbf{c} / \mathbf{2 c}$ with $\mathbf{2 h} / \mathbf{1 h}$, respectively, obtaining similar results than for the transformations shown in Scheme 2

[26] This is due to the fact that after $24 \mathrm{~h}$ the activity of LBADH and the effective concentration of $\mathrm{NADP}(\mathrm{H})$ considerably decrease (data not shown).

[27] 1010 Sherbrooke Street West, Suite 910, Montréal, Québec, Canada; http://www.chemcomp.com.

[28] J. Wang, P. Cieplak, P. A. Kollman, J. Comput. Chem. 2000, 21, 1049-1074.

[29] M. Wojciechowski, B. Lesyng, J. Phys. Chem. B 2004, 108, 18368-18376.

[30] For a discussion on the dielectric constant of a globular protein matrix, see: L. Monique, Biochim. Biophys. Acta Protein Struct. Mol. Enzymol. 1998, 1386 , 305-330.

[31] W. L. DeLano. The PyMOL molecular graphics system. DeLano Scientific 2002, San Carlos, CA, USA; http://www.pymol.org.

Received: ((will be filled in by the editorial staff))

Revised: ((will be filled in by the editorial staff)) Published online: ((will be filled in by the editorial staff)) 
Entry for the Table of Contents

Thermodynamics or Kinetics?

Fabricio R. Bisogno, Eduardo

García-Urdiales, Haydee Valdés,

Iván Lavandera, Wolfgang Kroutil,

Dimas Suárez, and Vicente Gotor* Page - Page

Ketone-Alcohol Hydrogen

Transfer Equilibria: Is the

Biooxidation of Halohydrins

Blocked?

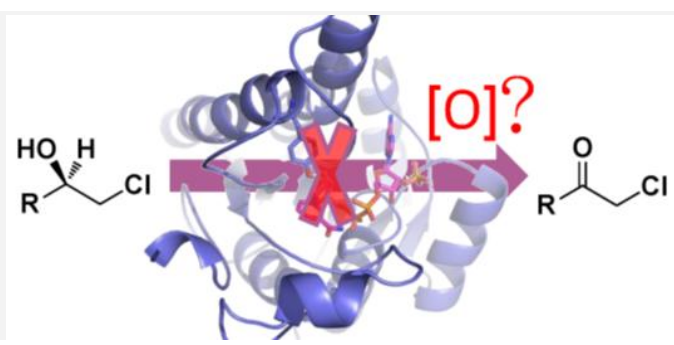

To provide a rationale to the quasi- Thermodynamics turns out to be the irreversibility of the HT-

bioreduction of $\alpha$-halo carbonyl

compounds, we study the redox

equilibrium of a series of ketones driving force of these processes.

combining experimental and theoretical elements.

Additionally, an IR-based method to predict the conversion of the HTreduction of a given ketone and a strategy to achieve the HT oxidation of halohydrins is also shown. 